\title{
Laparoscopically resected Castleman's disease in the pelvic retroperitoneum: A case report
}

\author{
KUMIKO NAKATA, NAOYUKI IWAHASHI, HITOMI MATSUKAWA, TOMOKO NOGUCHI, \\ TAMAKI YAHATA, NAMI OTA, YASUSHI MABUCHI and KAZUHIKO INO
}

Department of Obstetrics and Gynecology, Wakayama Medical University, Wakayama 641-0012, Japan

Received March 18, 2019; Accepted November 27, 2019

DOI: $10.3892 / \mathrm{mco} .2019 .1963$

\begin{abstract}
Castleman's disease is a rare benign disorder of unknown etiology characterized by proliferation of lymphoid tissues. Castleman's disease arising from pelvic retroperitoneum is clinically rare. The present case report describes a rare case of laparoscopically resected Castleman's disease in the pelvic retroperitoneum associated with benign ovarian cyst. A 47-year-old woman, gravida 5, para 3, was referred to to the Department of Obstetrics and Gynecology of Wakayama Medical University with a suspected pelvic tumor. Magnetic resonance imaging revealed that the solid tumor was localized in the retroperitoneal space at the right side of the pelvis. The patient underwent laparoscopic surgery for the resection of the pelvic retroperitoneal tumor, with complete tumor resection. Postoperative pathological examination established the diagnosis of Castleman's disease. The postoperative course was uneventful, with no evidence of local recurrence or systemic disease 6 months after diagnosis.
\end{abstract}

\section{Introduction}

Castleman's disease is a rare benign disorder of unknown etiology characterized by proliferation of lymphoid tissues (1). Although Castleman's disease has been found in lymphatic tissues of the chest, neck, abdomen, and pelvis, its retroperitoneal localization is rare; further, its localization in the pelvic retroperitoneum is extremely rare $(2,3)$. To date, only 14 cases of retroperitoneal localization of Castleman's disease occurring in female pelvis have been documented in the English literature (Table I) (4-17). The pre-operative diagnosis of pelvic retroperitoneal Castleman's disease is difficult, and most cases have been diagnosed based on post-operative pathological examination. Furthermore, due to the limited

Correspondence to: Dr Naoyuki Iwahashi, Department of Obstetrics and Gynecology, Wakayama Medical University, 811-1 Kimiidera, Wakayama 641-0012, Japan

E-mail: naoyuki@wakayama-med.ac.jp

Key words: Castleman's disease, retroperitoneal tumor, ovarian tumor, laparoscopic surgery number of cases described, the appropriate clinical management strategy for this disease is not yet established. Herein, we report a rare case of Castleman's disease localized in the female pelvic retroperitoneum, which was completely resected by laparoscopic surgery.

\section{Case report}

A 47-year-old gravida 5, para 3 female was referred to our hospital because of a pelvic mass accidentally found by radiological examination at a health checkup. She had no systemic symptoms such as pelvic pain, fatigue, fever, or weight loss. Transvaginal ultrasound examination revealed a $7.8 \mathrm{~cm}$-sized simple cystic tumor on the left ovary. Magnetic resonance imaging (MRI) revealed an $8.2 \mathrm{~cm}$-sized ovarian cyst and a $5.2 \mathrm{~cm}$-sized heterogeneously hyper-enhanced solid tumor in the retroperitoneal space of the left pelvis on T2-weighted (T2W) images (Fig. 1A). On T1-weighted (T1W) images, the pelvic retroperitoneal tumor was homogeneous and nearly isointense to uterus in signal intensity. Computed tomography (CT) revealed partial calcification of both the cystic and solid pelvic retroperitoneal tumors, with no pelvic lymph node swelling (Fig. 1B). ${ }^{18}$ F-fluoro-2-deoxy-D-glucose positron emission tomography combined with computed tomography (FDG-PET/CT) detected high radiotracer uptake by the retroperitoneal tumor [maximum standardized uptake value (SUVmax): 4.14], with no distant metastases (Fig. 1C). Serum levels of carcinoembryonic antigen (CEA), cancer antigen (CA) 19-9, CA125, and interleukin 6 (IL-6) were within the normal range. Except for the above-mentioned findings, serum biochemical and clotting studies were within the normal limits.

A laparoscopic surgery was performed for both pelvic retroperitoneal tumor and left ovarian cyst. Intraoperative examination evidenced that the pelvic retroperitoneal tumor was most likely to represent a lymph node enlargement at the left external iliac area and was associated with mobile and smooth left ovarian tumor (Fig. 2A). A laparoscopic monopolar device was applied to excise the pelvic peritoneum and expose the pelvic mass, and a bipolar device was subsequently applied to ensure coagulation around the tumor. Although it was closely located to the external iliac vessels, no direct vascular invasion was noted in the tumor and its surface was well circumscribed (Fig. 2B). This way, tissues around the tumor could be dissected and the feeding vessel 
desiccated from the external iliac artery with limited bleeding. No enlarged lymph nodes were found at the retroperitoneal region. These tumors were placed in a plastic bag and removed through the umbilical trocar site.

Macroscopically, the pelvic retroperitoneal tumor was rubbery firm, with the cut surface appearing soft, finely granular, and pale yellow in color (Fig. 2C). Microscopic examination revealed marked follicular hyperplasia with concentric layering of mantle-zone lymphocytes (Fig. 3). Most follicles were hyalinized and atrophic, with an expanded follicular dendritic cell network. Paraffin section immunostain showed mixed populations of CD20-immunoreactive B cells and $\mathrm{CD} 3$-immunoreactive $\mathrm{T}$ cells and negative staining for immunoglobulin G4 (IgG4) (data not shown). The pathological diagnosis was unicentric and hyaline vascular type of Castleman's disease localized in the pelvic retroperitoneum. The cystic tumor in the left ovary was diagnosed as a benign simple cyst. The postoperative course was uneventful, and the patient was discharged from the hospital on postoperative day 5. Six months after diagnosis, there was no evidence of local recurrence or systemic disease.

\section{Discussion}

The present case refers to an incidentally found unicentric and hyaline vascular type of Castleman's disease localized in the pelvic retroperitoneum, with no symptoms. Castleman's disease is a rare, benign lymphoproliferative disorder originally described by Castleman and Towne in 1954 (18). Castleman's disease includes unicentric and multicentric forms, which are thought to represent distinct clinical entities with different risk factors, presentation, treatment response, and long-term survival (19). The most common-unicentric-form, is often found in both males and females aged 20-30 years and presents asymptomatically or with compressive symptoms related to the mass (20). Unicentric Castleman's disease affects a single group of lymph nodes, often in the chest or abdomen, and is generally cured with surgical resection of the involved group of lymph nodes (19). Multicentric Castleman's disease is more often found in adults aged 50-60 years and is likely to present with systemic symptoms, including fever, malaise, night sweats, weakness, weight loss, and peripheral lymphadenopathy (20). Multicentric Castleman's disease may also be found in immunosuppressed patients infected with HIV and human herpesvirus 8 (21). Multicentric Castleman's disease tends to behave aggressively, similarly to a lymphoma, and is less likely to be cured by surgical resection (19). Castleman's disease can also be grouped into hyaline vascular and plasma cell types according to histopathological subsets (22). The hyaline vascular type accounts for $90 \%$ of cases. Recently, a mixed type of hyaline vascular and plasma cell types has also been reported (23).

Preoperative diagnosis of Castleman's disease-especially when located in the pelvis-is difficult due to its very low frequency and nonspecific radiographic signs. Benign retroperitoneal tumors are relatively uncommon, comprising only approximately $20 \%$ of all primary retroperitoneal neoplasms (24). The differential diagnosis of a retroperitoneal mass includes lymphoma, sarcoma, metastasis, neural tumor, and granulomatous disease (2). Among these entities, lymphoma is the most difficult to distinguish from Castleman's disease because of its similar homogeneity on radiographic findings. CT generally demonstrates a homogeneous soft tissue mass, and both histological types of Castleman's disease show contrast enhancement (25). Variable patterns of calcification can be seen in CT in up to $31 \%$ of cases, including the present one (25). On MRI, unicentric Castleman's disease exhibits mild hyperintensity in $\mathrm{T} 1 \mathrm{~W}$ and $\mathrm{T} 2 \mathrm{~W}$ imaging compared with skeletal muscle (26). MRI is particularly suitable to evaluate the extent of the lesion and its relationship to adjacent structures, but it is less sensitive to calcifications (2). In FDG-PET/CT, Castleman's disease demonstrates only moderate radiotracer uptake with reported SUVmax between 4.7 and 5.8 (27,28). Most active lymphomas express much higher average SUVs than FDG-avid lymph nodes in Castleman's disease $(29,30)$; however, some degree of overlap with low- and intermediate-grade lymphoma is possible. Murphy et al (30) emphasized that, in several cases of lowand intermediate-grade lymphomas, SUVs are higher than those found in Castleman's disease. Retroperitoneal sarcoma and lymphoma are more likely to demonstrate higher apparent diffusion coefficient values (ADC) on MRI (31) and higher ${ }^{18} \mathrm{~F}-\mathrm{FDG}$ avidity (SUVmax, 4.2-23.6) on PET/CT (32). In the present case, MRI allowed us to conclude that the mass was located on the retroperitoneal space and not in the ovary, and FDG-PET/CT helped to narrow down the differential diagnosis, particularly considering lymphoma and sarcoma.

In the English literature, a small number of Castleman's disease cases have been reported in the female pelvis (4-17), with clinical characteristics resembling a tubo-ovarian abscess, endometriotic cyst, or dermoid cyst of ovarian origin. The mean age and tumor size of Castleman's disease cases were $26.5(13-58)$ and $7.5 \mathrm{~cm}(5.0-10.0 \mathrm{~cm})$, respectively (Table I). Due to the low incidence of Castleman's disease in the female pelvis and the clinical resemblances with other pelvic masses, it may be difficult to diagnose Castleman's disease in initial evaluation and the case may be included in the differential diagnosis of pelvic mass. In most cases, pelvic Castleman's disease presents as a pelvic mass with clinical suspicion of adnexal tumor. Fortunately, this case of pelvic retroperitoneal Castleman's disease was associated with an ipsilateral benign ovarian cyst, which also helped to diagnose the mass as retroperitoneal-and not ovarian-in origin. Castleman's disease is an important consideration in the differential diagnosis of pelvic masses in women.

The appropriate clinical management strategy for pelvic retroperitoneal Castleman's disease has not been established, as clinical experience with such cases is limited. While only limited successful management with steroids and cytotoxic agents has been reported (33), surgical excision has been considered as standard therapy. A wide, complete excisional margin is preferred due to the lesion's infiltrative pattern and potential for recurrence. Incomplete resection may be associated with poorer outcome (34). Although Castleman's disease has a benign histology, surgical excision is not always easy. As observed in the present case, hypervascularity is frequently associated with massive hemorrhage at excision. Pelvic retroperitoneal Castleman's disease is often accompanied by remarkable fibrous adhesion to the surrounding tissues (35). To avoid massive hemorrhage, it is essential to fully dissect 
Table I. Previously reported cases of Castleman's disease in the female pelvic retroperitoneum since 1962 in English literature.

\begin{tabular}{|c|c|c|c|c|c|}
\hline Author, year & Age, years & Disease site & Tumor size & Treatment & (Refs.) \\
\hline Latters and Pachter, 1962 & 29 & Pre-sacral & $5.0 \times 2.5 \times 2.0 \mathrm{~cm}$ & Abdominal resection & (4) \\
\hline Giaretta et al, 1971 & 37 & Para-iliac vessel & $9.0 \mathrm{~cm}$ & Abdominal resection & $(5)$ \\
\hline Emson, 1973 & 14 & Para-iliac vessel & not specified & Abdominal biopsy & (6) \\
\hline Bainbridge 1976 & 21 & Para-iliac vessel & $8.0 \times 5.0 \times 4.0 \mathrm{~cm}$ & Abdominal resection & (7) \\
\hline Tanaka et al, 1976 & 36 & Para-iliac vessel & $9.0 \times 8.0 \times 5.0 \mathrm{~cm}$ & Abdominal resection & (8) \\
\hline Kumar and Shah, 1979 & 19 & Adherent to ovary & $10.0 \times 9.0 \times 3.0 \mathrm{~cm}$ & Abdominal resection & (9) \\
\hline Goodman et al, 1983 & 17 & Pre-sacral & $8.0 \times 5.0 \times 4.0 \mathrm{~cm}$ & Abdominal resection & $(10)$ \\
\hline Ylinen et al, 1995 & 26 & Retro pubic & $5.0 \times 4.0 \times 4.0 \mathrm{~cm}$ & Abdominal resection & (11) \\
\hline MacDonald et al, 1996 & 58 & Sigmoid colon mesentery & $8.0 \times 4.0 \mathrm{~cm}$ & Abdominal resection & (12) \\
\hline Gaunt et al, 2002 & Not specified & Pre-sacral & $6.0 \mathrm{~cm}$ & Abdominal resection & (13) \\
\hline Nakamura et al, 2004 & 30 & Para-iliac vessel & $6.5 \times 5.0 \times 4.0 \mathrm{~cm}$ & Abdominal resection & (14) \\
\hline Sato, 2013 & 22 & Para-sacral vessel & $9.5 \times 7.0 \times 7.0 \mathrm{~cm}$ & Abdominal resection & $(15)$ \\
\hline Lee et al, 2015 & 27 & Para-iliac vessel & $7.0 \mathrm{~cm}$ & Laparoscopic resection & (16) \\
\hline Schelble and Merritt, 2019 & 13 & Pelvic side wall & $5.5 \times 3.5 \times 2.0 \mathrm{~cm}$ & Abdominal resection & $(17)$ \\
\hline Present study & 47 & Para-iliac vessel & $5.2 \mathrm{~cm}$ & Laparoscopic resection & - \\
\hline
\end{tabular}
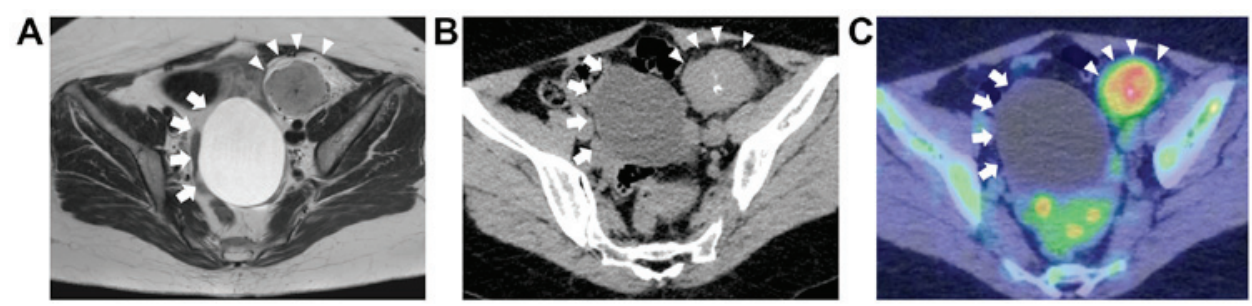

Figure 1. Radiological findings of pelvic retroperitoneal Castleman's disease. Arrowheads indicate retroperitoneal tumor. Arrows indicate ovarian cyst. (A) T2-weighted magnetic resonance imaging findings. (B) Computed tomography findings. (C) ${ }^{18} \mathrm{~F}$-fluoro-2-deoxy-D-glucose positron emission tomography combined with computed tomography findings.
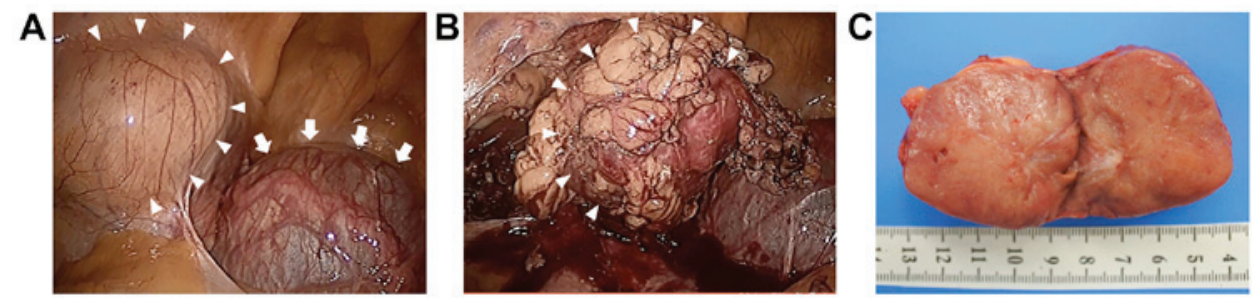

Figure 2. Laparoscopic and macroscopic findings of pelvic retroperitoneal Castleman's disease. Arrowheads indicate retroperitoneal tumor. Arrows indicate ovarian cyst. (A) Left retroperitoneal tumor and left ovarian tumor. (B) Retroperitoneal tumor surface. (C) Cut surface of retroperitoneal tumor macroscopic findings.
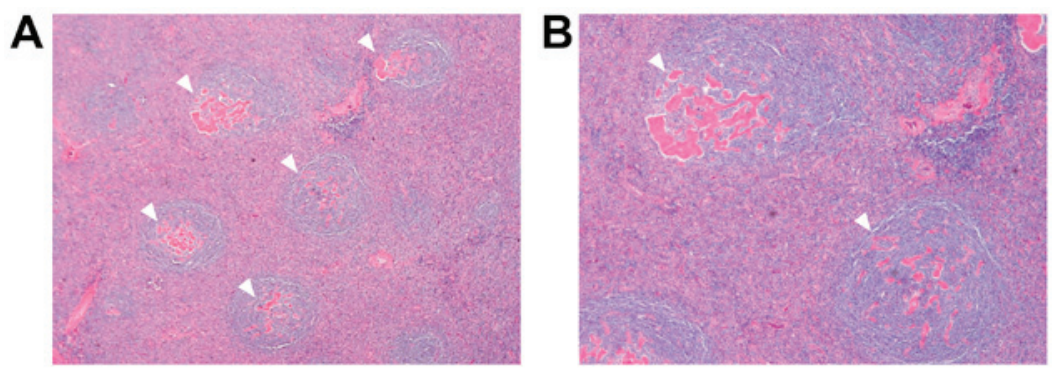

Figure 3. Microscopic findings of pelvic retroperitoneal Castleman's disease. Hematoxylin and eosin staining. (A) Magnification, x4; and (B) magnification, x20. Arrowheads indicate hyalinization. 
tissues around the tumor and have enough space to desiccate the feeding vessel originated from large vessel. A thorough pre-operative discussion about radiological examination could be useful for assisting the preparation for surgical resection. Although several cases of unicentric Castleman's disease in the abdominal cavity treated laparoscopically have been reported $(36,37)$, to the best of our knowledge, only one report on female pelvic retroperitoneal Castleman's disease treated by laparoscopy is available in literature (Table I) (16). As some masses are well vascularized and adjacent to the great vessels, laparoscopy can provide magnified images that facilitate and secure dissection.

In summary, a rare case of female pelvic retroperitoneal Castleman's disease was found, for which a laparoscopic surgery was performed. Gynecologists should be aware of the possible event of such rare cases, and available surgical interventions should be fully discussed. Laparoscopic surgery may be a possible treatment option for such a rare condition.

\section{Acknowledgements}

Not applicable.

\section{Funding}

No funding was received.

\section{Availability of data and materials}

The datasets uesd and/or analyzed during the current study are available from the corresponding author on reasonable request.

\section{Author's contributions}

$\mathrm{KN}$, NI and KI conceived and designed this case report. KN, NI and KI wrote the initial draft of the report. HM, TN and TY acquired the data in the surgical field. NO and YM acquired the data in the diagnostic imaging and pathological examination. All authors have read and approved the final version of the manuscript.

\section{Ethics approval and consent to participate}

Written informed consent for surgery was obtained from the patient.

\section{Patient consent for publication}

Written informed consent for publication of the present report was obtained from the patient.

\section{Competing interests}

The authors declare that they have no competing interests.

\section{References}

1. Castleman B, Iverson L and Menendez VP: Localized mediastinal lymph node hyperplasia resembling thymoma. Cancer 9: 822-830, 1956.
2. Bonekamp D, Horton KM, Hruban RH and Fishman EK: Castleman disease: The great mimic. Radiographics 31: 1793-1807, 2011

3. Aygun C, Tekin MI, Demirhan B, Peskircioglu CL, Agildere M and Ozkardes H: A case of incidentally detected Castleman's disease with retroperitoneal paravertebral localization. Int J Urol 7: 22-25, 2000.

4. Latters R and Pachter MR: Benign lymphoid masses of probable hamartomatous nature. Analysis of 12 cases. Cancer 15: 197-214, 1962.

5. Giaretta MI, Hyun J and Gibbons JM Jr: Angiomatous lymphoid hamartoma as a pelvic mass. A case report. Obstet Gynecol 38: 391-394, 1971.

6. Emson HE: Extrathoracic angiofollicular lymphoid hyperplasia with coincidental myasthenia gravis. Cancer 31: 241-245, 1973.

7. Bainbridge ET: Angiomatous lymphoid hamartoma of the pelvis Br J Obstet Gynaecol 83: 823-826, 1976.

8. Tanaka T, Kobayashi K, Sho T and Hamazaki M: Castleman's lymphoma among Japanese population. Acta Pathol Jpn 26: 547-559, 1976.

9. Kumar D and Shah S: Angiomatous lymphoid hamartoma or pseudolymphoma of pelvic retroperitoneum: Clinical significance and evaluation of pelvic ureter. Urology 13: 677-681, 1979.

10. Goodman K, Baim RS, Clair MR and Perkes EA: Angiomatous lymphoid hamartoma of the pelvis. Characteristic calcification and computed tomographic appearance. Radiology 146: 728, 1983.

11. Ylinen K, Sarlomo-Rikala $M$ and Laatikainen T: Pelvic Castleman disease mimicking an adnexal tumor. Obstet Gynecol 85: 894-897, 1995.

12. MacDonald SR, Lurain JR, Hoff F, Variakojis D and Fishman DA: Castleman disease presenting as a pelvic mass. Obstet Gynecol 87: 875-877, 1996.

13. Gaunt GA, Gostout BS, Remstein E and Cliby WA: Pelvic Castleman disease presenting as vaginal occlusion. Obstet Gynecol 100: 1082-1085, 2002.

14. Nakamura Y, Tokuyama O, Muso A, Kawamura N, Yasui T and Ishiko O: Asymptomatic pelvic Castleman disease in an infertile woman: Case report. Arch Gynecol Obstet 269: 156-158, 2004.

15. Sato A: Castleman's disease in the pelvic retroperitoneum: A case report and review of the Japanese literature. Int J Surg Case Rep 4: 19-22, 2013.

16. Lee J, Paek J, Lee YH, Kong TW, Chang SJ and Ryu HS: Pelvic Castleman's disease presenting as an adnexal tumor in a young woman. Obstet Gynecol Sci 58: 323-326, 2015.

17. Schelble AP and Merritt DF: Pelvic Castleman's disease presenting as an adnexal mass in an adolescent. J Pediatr Adolesc Gynecol 32: 86-89, 2019.

18. Castleman B and Towne VW: Case records of the Massachusetts General Hospital: Case No. 40231. N Engl J Med 250: 1001-1005, 1954.

19. Talat N, Belgaumkar AP and Schulte KM: Surgery in Castleman's disease: A systematic review of 404 published cases. Ann Surg 255: 677-684, 2012.

20. Roca B: Castleman's disease. A review. AIDS Rev 11: 3-7, 2009.

21. Shah D, Darji P, Lodha S and Bolla S: Unicentric Castleman's disease of abdomen. J Radiol Case Rep 7: 26-33, 2013.

22. Keller AR, Hochholzer L and Castleman B: Hyaline-vascular and plasma-cell types of giant lymph node hyperplasia of the mediastinum and other locations. Cancer 29: 670-683, 1972.

23. Seco JL, Velasco F, Manuel JS, Serrano SR, Tomas L and Velasco A: Retroperitoneal Castleman's disease. Surgery 112: 850-855, 1992.

24. Takihara H, Yamakawa G, Baba Y, Takahashi M and Ishihara T: Castleman disease. Unusual retroperitoneal location indistinguishable from malignant tumor in preoperative angiographic appearance. Urology 41: 162-164, 1993.

25. Chung EM, Biko DM, Arzamendi AM, Meldrum JT and Stocker JT: Solid tumors of the peritoneum, omentum, and mesentery in children: radiologic-pathologic correlation: From the radiologic pathology archives. Radiographics 35: 521-546, 2015.

26. Ko SF, Hsieh MJ, Ng SH, Lin JW, Wan YL, Lee TY, Chen WJ and Chen MC: Imaging spectrum of Castleman's disease. AJR Am J Roentgenol 182: 769-775, 2004.

27. Hill AJ, Tirumani SH, Rosenthal MH, Shinagare AB, Carrasco RD, Munshi NC, Ramaiya NH and Howard SA: Multimodality imaging and clinical features in Castleman disease: Single institute experience in 30 patients. Br J Radiol 88: 20140670, 2015 
28. Lee ES, Paeng JC, Park CM, Chang W, Lee WW, Kang KW, Chung JK and Lee DS: Metabolic characteristics of Castleman disease on 18F-FDG PET in relation to clinical implication. Clin Nucl Med 38: 339-342, 2013.

29. Oida Y, Shimizu K, Mukai M, Imaizumi T, Nakamura M and Makuuchi H: FDG-PET and diffusion-weighted MR imaging appearance in retroperitoneal Castleman's disease: A case report. Clin Imaging 32: 144-146, 2008.

30. Murphy SP, Nathan MA and Karwal MW: FDG-PET appearance of pelvic Castleman's disease. J Nucl Med 38: 1211-1212, 1997.

31. Nakayama T, Yoshimitsu K, Irie H, Aibe H, Tajima $T$, Shinozaki K, Nishie A, Asayama Y, Kakihara D, Matsuura S and Honda $\mathrm{H}$ : Usefulness of the calculated apparent diffusion coefficient value in the differential diagnosis of retroperitoneal masses. J Magn Reson Imaging 20: 735-742, 2004.

32. Platzek I, Beuthien-Baumann B, Schramm G, Maus J, Laniado M, Kotzerke J, van den Hoff J and Schuler M: FDG PET/MR in initial staging of sarcoma: Initial experience and comparison with conventional imaging. Clin Imaging 42: 126-132, 2017.

33. Summerfield GP, Taylor W, Bellingham AJ and Goldsmith HJ: Hyaline-vascular variant of angiofollicular lymph node hyperplasia with systemic manifestations and response to corticosteroids. J Clin Pathol 36: 1005-1011, 1983.
34. Bowne WB, Lewis JJ, Filippa DA, Niesvizky R, Brooks AD, Burt ME and Brennan MF: The management of unicentric and multicentric Castleman's disease: A report of 16 cases and a review of the literature. Cancer 85: 706-717, 1999.

35. Kiguchi H, Ishii T, Ishikawa Y, Masuda S, Asuwa N, Yamafuji K and Takahashi T: Castleman's disease of the abdomen and pelvis: Report of three cases and a review of the literature. J Gastroenterol 30: 661-666, 1995.

36. Corcione F, Caiazzo P, Cuccurullo D, Settembre A, Miranda L, Pirozzi F and Caracino V: Laparoscopic treatment of unicentric Castleman's disease with abdominal localization. J Laparoendosc Adv Surg Tech A 15: 400-404, 2005.

37. Brusciano L, Rossetti G, Maffettone V, Napolitano V, Izzo D, Pizza F Russo G, Russo F, del Genio G and del Genio A: Laparoscopic treatment of an uncommon abdominal localization of Castleman disease. Surg Laparosc Endosc Percutan Tech 15: 241-243, 2005.

This work is licensed under a Creative Commons Attribution-NonCommercial-NoDerivatives 4.0 International (CC BY-NC-ND 4.0) License. 